\title{
Inefficiencies in Public Electricity Provision and Impacts on Firms in Karachi's Manufacturing Sector
}

\begin{abstract}
NAUSHEEN H. ANWAR
The private costs of electricity supply failures are substantial and inimical to industrial productivity. Using results from a small sample survey of manufacturing firms in Karachi, the study documented the causes, extent and incidence of the failures, identified and classified the firms' private responses, and estimated the capital share of internally produced power and the associated costs. The results are reported here to engender discussion for developing a policy model of infrastructure provision suited to a developing country like Pakistan. The most encouraging options are those that allow for cooperative provision amongst firms with concurrent reforms in the regulatory and institutional environments. An optimal policy will allow inter-firm trading of electricity making the power market competitive. Those firms that already have extensive private generating capacity due to weak public supply will realise scale economies by selling electric power to lower the costs of private provision. Competition in electricity supply implies that industrial users will find attractive substitutes in the private sector. This will lead to a reduction in the demand on public service, already limited in quantity and quality in key urban-industrial locations like Karachi.
\end{abstract}

\section{INTRODUCTION}

Manufacturing firms in Karachi are investing in electricity supply for their own use. This is happening because publicly supplied electricity is of poor quality and subject to chronic failures. In Pakistan, public electricity provision suffers from two kinds of inefficiencies; (a) a non-performing public sector and (b) private provision by end users, specifically manufacturing firms. Pasha et al. (1990) observed the extensive existence and impact of such inefficiencies. The understanding is that private investment in electricity generation absorbs significant resources that could be otherwise allocated for efficient industrial production. Furthermore, small sized firms sustain the highest burden as they have a lower capacity to bear the capital costs of private power generation.

Nausheen H. Anwar is a Ph.D. candidate, Division of Urban Planning, Columbia University, New York, USA

Author's Note: I wish to acknowledge the invaluable assistance of Professor Haroon Jamal in performing the econometric analysis. Prof. Jamal is Senior Economist at the Applied Economics Research Centre, Karachi University, Karachi. 
In Karachi several firms in a variety of manufacturing sectors have invested in electricity generation. They rely mainly on the publicly available service but supplement it with private provision or vice versa. The extent, incidence and costs of private substitution vary by industrial sector and by firm size. Notably, the private provision of electricity is subject to a high degree of scale economies; the average cost per unit of quantity produced falls with increases in the total quantity produced.

The objective of this paper is threefold. First, it is highlighted how deficiencies in electricity supply affect manufacturing firms. Second, it describes how firms respond to these deficiencies and estimates the costs of the responses, as well as the extent of private costs as a measure of firms' willingness to pay for a reliable service. Third, based on empirical observations, the study offers policy options that delineate alternative institutional arrangements for electricity provision without advocating an outright replacement of the public sector. It posits that current reforms, such as privatisation, necessitate extensive capital outlays and the establishment of well-functioning contractual frameworks, currently not present in Pakistan. Consequently, there is a need to address the inefficiency problem in a way that minimises costs for the industrial sector in the intermediate period.

The conceptual framework of this discussion is based on recent important theoretical and empirical literature [Sclar (2000); Vickers and Yarow (1997); Batten (1996); Mody (1996); Pollitt (1995); Clark and Pitelis (1994); Kessides (1993); Baumol and Lee (1991)]. The framework emphasises that optimal policies for promoting efficiency and equity in infrastructure provision are predicted on fostering competition through regulatory and institutional reforms. Such reforms should endeavour to create an appropriate public-private balance in infrastructure provision, without banishing the public sector from the economic scene.

\section{THE CAUSES OF INEFFICIENCY IN ELECTRICITY SUPPLY}

It is common knowledge that manufacturing firms in Pakistan suffer from frequent interruptions in public electricity service forcing most to undertake extra investments in generators to avoid production losses and damage to machinery and equipment. Although public utilities like WAPDA and KESC have extensive capital investment in electric power production, they fail to deliver service at the required level of demand. The failures are a result of the low ratio of available capacity to installed capacity, extensive transmission and distribution (T\&D) losses, and nontechnological or $x$-inefficiency factors.

In Karachi power interruptions are due to bottlenecks and congestion on the ailing $T \& D$ networks as well as $\mathrm{x}$-inefficiency factors concerning institutional weaknesses. The survey revealed that in Karachi's industrial areas, like SITE and Korangi, when large energy intensive manufacturing plants start to operate, the resulting voltage surge often damages the machinery and equipment of smaller 
neighbouring firms. Anecdotal evidence suggests that large energy intensive firms put a heavier load on the public system and tie up more operable transmission capacity. Within the KESC network the congestion is sometimes so severe that the system fails completely leading to prolonged power outages in industrial areas. The important observation here is that congestion in the use of the system is an additional factor that promotes T\&D losses. While treating congestion as endogenous is common in levying tariffs in infrastructure sectors like transportation, public agencies in developing countries like Pakistan have not understood the critical role this factor plays in determining optimal pricing policy for electricity service. They will have to consider designing specific tariff systems that set a congestion toll reducing the load and congestion to a socially optimal level. This will encompass a comprehensive study of KESC's costs and variations in demand by end-user types and locations to determine the order of the price that removes congestion. This is critical in the short run when deep capital and institutional constraints exist on system expansion.

In the past decades, KESC's T\&D losses have steadily grown to a staggering 35 percent level, a figure that is extremely high in comparison to other Asian urban electric utilities. For instance, the Hong Kong HEC experiences only 12 percent T\&D losses and Philippines' MERALCO registers approximately 16 percent. The KESC's T\&D losses are due to (i) frequent overloading of old transmission lines and distribution feeders, (ii) congestion in the use of the system, and (iii) theft of power and unmetered supply that drain energy. Given that Karachi is the economic centre of Pakistan, the electricity demand in the KESC supply area has grown exponentially. But policy-makers have been negligent inaccurately forecasting demand. KESC also lacks the institutional autonomy needed to implement rational policy for reforming and expanding the system. Currently, the central government is planning to subject utilities like KESC to market discipline through privatisation. While this is laudable, there remain critical $\mathrm{x}$-inefficiency factors that will hamper such efforts, and need to be carefully addressed before privatisation. The labour unions and political groups that are well-entrenched in its administrative structure, have vested interests in maintaining the status quo. Furthermore, reforms in public sector performance need political consensus between the central and provincial levels of decision-making. A key obstacle is Pakistan's ad hoc regulatory system and it's deteriorating institutional environment, which creates incentives for misappropriation of resources. Any successful reform in heavy investment infrastructure sectors is dependent on the existence of institutions that protect such investments and engender respect for property rights. For instance, the successful privatisation of the electricity sector in countries like Chile occurred because market reform was preceded by major transformations of the regulatory and institutional environments. Significantly, there also existed in Chile a strong will for privatisation whereby ordinary public sector employees perceived themselves to have a positive stake 
in reform. In Pakistan, such facilitating characteristics are not currently present in the public sector. Therefore, inefficiencies in electricity provision will not be mitigated through further investments in new facilities or by changing pricing policies, or even by implementing privatisation.

\section{THE EXTENT AND INCIDENCE OF THE INEFFICIENCIES}

The survey was based on a pre-designed questionnaire of a stratified random sample of 150 manufacturing firms located in Karachi. Information on the population of firms was obtained from the provincial directory of industry prepared by the Sindh Chamber of Commerce, and information published by industrial trade associations. A low completion rate of 50 percent was due to Karachi's law and order situation that impeded data collection. Therefore the analysis is based on those firms that successfully completed the questionnaire. Tables $1.1-1.8$ show the general characteristics of the realised sample and the variations in the incidence of failures. The year chosen for analysis is 1997 and the firms are classified into 12 industrial groups listed by employment size in Table 1. Results of the study are discussed below.

The survey results reveal large variations in the availability and quality of publicly provided electricity and in the firms' behaviour across all industrial sectors. These variations should be taken into account in government reforms for the electricity sector. Table 1.1 (Distribution of Manufacturing Firms by Area and Sources of Electricity), shows that only 17 out of the 73 firms or 23 percent do not have private electricity generators. This implies that the lack of reliable electricity supply is quite a serious problem in Karachi's industrial sector. Table 1.2 (Distribution of Manufacturing Firms: Sources of Electricity by Firm Size), shows that a significant number of captive firms are generally small in size, e.g., 1-49 employees. Table 1.3 (Distribution of Power Outages by Firm Size), illustrates that small firms also are subject to a high number of power failures, although larger firms within the 200-499 employee category also suffer the bulk of outages. Interviews with the targeted 150 firms support the observation that small firms tend not to have private generating equipment. This occurs because small firms are unable to achieve economies of scale in private electricity generation. Table 1.1 also shows that there are no significant variations in the quality of public provision by industrial area, supporting the observation that inefficiencies are present in all the industrial areas of Karachi.

Table 1.4 (Working Hours Lost Due to Power Outage by Firm Size), clearly shows that nearly 75 percent of the surveyed firms lost in excess of 100 working hours in 1997 due to power outage, and approximately 25 percent of these were small sized firms. Table 1.5 (Production Shut-down During Power Failure by Firm Size), shows that nearly 50 percent of the firms surveyed experienced production 
Table 1

Distribution of Manufacturing Firms by Industry and Employment Size

\begin{tabular}{|c|c|c|c|c|c|c|c|}
\hline \multirow[b]{2}{*}{ Industry } & \multicolumn{6}{|c|}{ Establishment Employment Size } & \multirow[b]{2}{*}{ Total } \\
\hline & $1-49$ & $50-99$ & $100-199$ & $200-499$ & $500-999$ & $1000+$ & \\
\hline \multirow{3}{*}{$\begin{array}{l}\text { Food and } \\
\text { Beverage }\end{array}$} & $(0)$ & $(0)$ & $(0)$ & (1) & $(0)$ & $(0)$ & (1) \\
\hline & .00 & .00 & .00 & 100.00 & .00 & .00 & 100.00 \\
\hline & .00 & .00 & .00 & 3.85 & .00 & .00 & 1.37 \\
\hline \multirow{3}{*}{$\begin{array}{l}\text { Textile } \\
\text { Products }\end{array}$} & (1) & (1) & (1) & (5) & (3) & (3) & (14) \\
\hline & 7.14 & 7.14 & 7.14 & 35.71 & 21.43 & 21.43 & 100.00 \\
\hline & 6.67 & 16.67 & 10.00 & 19.23 & 27.27 & 60.00 & 19.00 \\
\hline \multirow{3}{*}{$\begin{array}{l}\text { Apparel and } \\
\text { Other }\end{array}$} & (4) & (1) & (2) & (2) & (2) & (0) & (11) \\
\hline & 36.36 & 9.09 & 18.18 & 18.18 & 18.18 & .00 & 100.00 \\
\hline & 26.67 & 16.67 & 20.00 & 7.69 & 18.18 & .00 & 15.07 \\
\hline \multirow{3}{*}{$\begin{array}{l}\text { Furniture and } \\
\text { Fixtures }\end{array}$} & (3) & $(0)$ & $(0)$ & (0) & (0) & (0) & (3) \\
\hline & 100.00 & .00 & .00 & .00 & .00 & .00 & 100.00 \\
\hline & 20.00 & .00 & .00 & .00 & .00 & .00 & 4.11 \\
\hline \multirow{3}{*}{$\begin{array}{l}\text { Chemical and } \\
\text { Pharma }\end{array}$} & (5) & (1) & (4) & (5) & (3) & (0) & (18) \\
\hline & 27.78 & 5.56 & 22.22 & 27.78 & 16.67 & .00 & 100.00 \\
\hline & 33.33 & 16.67 & 40.00 & 19.23 & 27.27 & .00 & 24.66 \\
\hline \multirow{3}{*}{$\begin{array}{l}\text { Petroleum and } \\
\text { Coal }\end{array}$} & (0) & (1) & (1) & (1) & (0) & (0) & (3) \\
\hline & .00 & 33.33 & 33.33 & 33.33 & .00 & .00 & 100.00 \\
\hline & .00 & 16.67 & 10.00 & 3.85 & .00 & .00 & 4.11 \\
\hline \multirow{3}{*}{$\begin{array}{l}\text { Leather and } \\
\text { Other }\end{array}$} & (1) & (0) & (0) & (3) & (0) & (0) & (4) \\
\hline & 25.00 & .00 & .00 & 75.00 & .00 & .00 & 100.00 \\
\hline & 6.67 & .00 & .00 & 11.54 & .00 & .00 & 5.48 \\
\hline \multirow{3}{*}{$\begin{array}{l}\text { Fabricated } \\
\text { Metal }\end{array}$} & (0) & (0) & (1) & (1) & (1) & (0) & (3) \\
\hline & .00 & .00 & 33.33 & 33.33 & 33.33 & .00 & 100.00 \\
\hline & .00 & .00 & 10.00 & 3.85 & 9.09 & .00 & 4.11 \\
\hline \multirow{3}{*}{$\begin{array}{l}\text { Industrial } \\
\text { Machinery }\end{array}$} & (0) & (0) & (1) & (2) & (0) & (1) & (4) \\
\hline & .00 & .00 & 25.00 & 50.00 & .00 & 25.00 & 100.00 \\
\hline & .00 & .00 & 10.00 & 7.69 & .00 & 20.00 & 5.48 \\
\hline \multirow{3}{*}{$\begin{array}{l}\text { Electrical } \\
\text { Products }\end{array}$} & (1) & (1) & (0) & (4) & (0) & (0) & (6) \\
\hline & 16.67 & 16.67 & .00 & 66.67 & .00 & .00 & 100.00 \\
\hline & 6.67 & 16.67 & .00 & 15.38 & .00 & .00 & 8.22 \\
\hline \multirow{3}{*}{$\begin{array}{l}\text { Transport } \\
\text { Equipment }\end{array}$} & (0) & (0) & (0) & (0) & (2) & (1) & (3) \\
\hline & .00 & .00 & .00 & .00 & 66.67 & 33.33 & 100.00 \\
\hline & .00 & .00 & .00 & .00 & 18.18 & 20.00 & 4.11 \\
\hline \multirow[t]{3}{*}{ Other } & (0) & (1) & (0) & (2) & (0) & (0) & (3) \\
\hline & .00 & 33.33 & .00 & 66.67 & .00 & .00 & 100.00 \\
\hline & .00 & 33.33 & .00 & 7.69 & .00 & .00 & 4.11 \\
\hline \multirow[t]{3}{*}{ Total } & (15) & (6) & (10) & (26) & (11) & (5) & (73) \\
\hline & 20.55 & 8.22 & 13.70 & 35.62 & 15.07 & 6.85 & 100.00 \\
\hline & 100.00 & 100.00 & 100.00 & 100.00 & 100.00 & 100.00 & 100.00 \\
\hline
\end{tabular}


Table 1.1

Distribution of Manufacturing Firms by Area and Sources of Electricity**

\begin{tabular}{lccccc}
\hline Industrial & \multicolumn{3}{c}{ Sources of Electricity for Production } & \\
\cline { 2 - 5 } Area & KESC Only & KESC Main & OWN Main & OWN Only & Total \\
\hline Site & $(3)$ & $(23)$ & $(4)$ & $(1)$ & $(31)$ \\
& 9.68 & 74.19 & 12.90 & 3.23 & 100.00 \\
Korangi & 17.65 & 54.76 & 57.14 & 14.29 & 42.47 \\
& $(5)$ & $(11)$ & $(1)$ & $(2)$ & $(19)$ \\
& 26.32 & 57.89 & 5.26 & 10.53 & 100.00 \\
Federal B & 29.41 & 26.19 & 14.29 & 28.57 & 26.03 \\
& $(4)$ & $(1)$ & $(0)$ & $(0)$ & $(5)$ \\
& 80.00 & 20.00 & .00 & .00 & 100.00 \\
Landhi & 23.53 & 2.38 & .00 & .00 & 6.85 \\
& $(3)$ & $(6)$ & $(1)$ & $(3)$ & $(13)$ \\
& 23.08 & 46.15 & 7.69 & 23.08 & 100.00 \\
West Wharf & 17.65 & 14.29 & 14.29 & 42.86 & 17.81 \\
& $(2)$ & $(1)$ & $(1)$ & $(1)$ & $(5)$ \\
& 40.00 & 20.00 & 20.00 & 20.00 & 100.00 \\
Total & 11.76 & 2.38 & 14.29 & 14.29 & 6.85 \\
& $(17)$ & $(42)$ & $(7)$ & $(7)$ & $(73)$ \\
& 23.29 & 57.53 & 9.59 & 9.59 & 100.00 \\
& 100.00 & 100.00 & 100.00 & 100.00 & 100.00 \\
\hline
\end{tabular}

**KESC Only = Firm uses electricity 100 percent from KESC supply.

**KESC Main $=$ Firm uses KESC as main source of electricity supply and private generators as standby.

**OWN Main $=$ Firm uses KESC supply as standby.

**OWN Only $=$ Firm uses electricity supply 100 percent from private generators.

Table 1.2

Distribution of Manufacturing Firms: Sources of Electricity by Firm Size

\begin{tabular}{lccccccc}
\hline \multirow{2}{*}{$\begin{array}{l}\text { Electricity } \\
\text { Sources }\end{array}$} & $1-49$ & $50-99$ & $100-199$ & $200-499$ & $500-999$ & $1000+$ & Total \\
\cline { 2 - 6 } KESC Only & $(7)$ & $(1)$ & $(0)$ & $(6)$ & $(3)$ & $(0)$ & $(17)$ \\
& 41.18 & 5.88 & .00 & 35.29 & 17.65 & .00 & 100.00 \\
& 46.67 & 16.67 & .00 & 23.08 & 27.27 & .00 & 23.29 \\
KESC Main & $(6)$ & $(3)$ & $(10)$ & $(16)$ & $(5)$ & $(2)$ & $(42)$ \\
& 14.29 & 7.14 & 23.81 & 38.10 & 11.90 & 4.76 & 100.00 \\
& 40.00 & 50.00 & 100.00 & 61.54 & 45.45 & 40.00 & 57.53 \\
OWN Main & $(1)$ & $(2)$ & $(0)$ & $(2)$ & $(2)$ & $(0)$ & $(7)$ \\
& 14.29 & 28.57 & .00 & 28.57 & 28.57 & .00 & 100.00 \\
& 5.57 & 33.33 & .00 & 7.69 & 18.18 & .00 & 9.59 \\
OWN Only & $(1)$ & $(0)$ & $(0)$ & $(2)$ & $(1)$ & $(3)$ & $(7)$ \\
& 14.29 & .00 & .00 & 28.57 & 14.29 & 2.86 & 100.00 \\
& 6.67 & .00 & .00 & 7.69 & 9.09 & 60.00 & 9.59 \\
Total & $(15)$ & $(6)$ & $(10)$ & $(26)$ & $(11)$ & $(5)$ & $(73)$ \\
& 20.55 & 8.22 & 13.70 & 35.62 & 15.07 & .85 & 100.00 \\
& 100.00 & 100.00 & 100.00 & 100.00 & 100.00 & 100.00 & 100.00 \\
\hline
\end{tabular}


Table 1.3

Distribution of Manufacturing Firms: Power Outages by

Firm Size (Per Week in 1997)

\begin{tabular}{|c|c|c|c|c|c|c|c|}
\hline \multirow{2}{*}{$\begin{array}{l}\text { Power } \\
\text { Outage Times }\end{array}$} & \multicolumn{6}{|c|}{ Establishment Employment Size } & \multirow[b]{2}{*}{ Total } \\
\hline & $1-49$ & $50-99$ & $100-199$ & $200-499$ & $500-999$ & $1000+$ & \\
\hline $0-10$ & (3) & (2) & (2) & (9) & (4) & (4) & $(24)$ \\
\hline \multirow[t]{2}{*}{ Per/Week } & 12.50 & 8.33 & 8.33 & 37.50 & 16.67 & 16.67 & 100.00 \\
\hline & 20.00 & 33.33 & 20.00 & 34.62 & 36.36 & 80.00 & 32.88 \\
\hline $10-20$ & (10) & (1) & (2) & (4) & (4) & (1) & (14) \\
\hline \multirow[t]{2}{*}{ Per/Week } & 14.29 & 7.14 & 14.29 & 28.57 & 28.57 & 7.14 & 100.00 \\
\hline & 13.33 & 16.67 & 20.00 & 15.38 & 36.36 & 20.00 & 19.18 \\
\hline 20-HI & $(10)$ & (3) & (6) & (13) & (3) & (0) & $(35)$ \\
\hline \multirow[t]{2}{*}{ Per/Week } & 28.57 & 8.57 & 17.14 & 37.14 & 8.57 & .00 & 100.00 \\
\hline & 66.67 & 50.00 & 60.00 & 50.00 & 27.27 & .00 & 47.95 \\
\hline \multirow[t]{3}{*}{ Total } & (15) & (6) & (10) & (26) & (11) & (5) & (73) \\
\hline & 20.55 & 8.22 & 13.70 & 35.62 & 15.07 & 6.85 & 100.00 \\
\hline & 100.00 & 100.00 & 100.00 & 100.00 & 100.00 & 100.00 & 100.00 \\
\hline
\end{tabular}

Table 1.4

Working Hours Lost Due to Power Outage by Firm Size

\begin{tabular}{|c|c|c|c|c|c|c|c|}
\hline \multirow{2}{*}{$\begin{array}{l}\text { Hours Lost in } \\
1997\end{array}$} & \multicolumn{6}{|c|}{ Establishment Employment Size } & \multirow[b]{2}{*}{ Total } \\
\hline & $1-49$ & $50-99$ & $100-199$ & $200-499$ & $500-999$ & $1000+$ & \\
\hline \multirow[t]{3}{*}{ Zero Hours } & (3) & (1) & $(0)$ & (6) & (4) & (3) & $(17)$ \\
\hline & 17.65 & 5.88 & .00 & 35.29 & 23.53 & 17.65 & 100.00 \\
\hline & 20.00 & 16.67 & .00 & 23.08 & 36.36 & 60.00 & 23.29 \\
\hline \multirow[t]{3}{*}{ 100-199 Hours } & (1) & (2) & (2) & (3) & (0) & (0) & (8) \\
\hline & 12.50 & 25.00 & 25.00 & 37.50 & .00 & .00 & 100.00 \\
\hline & 6.67 & 33.33 & 20.00 & 11.54 & .00 & .00 & 10.96 \\
\hline \multirow[t]{3}{*}{ 200-499 Hours } & (5) & (0) & (2) & (9) & (2) & (1) & (19) \\
\hline & 26.32 & .00 & 10.53 & 47.37 & 10.53 & 5.26 & 100.00 \\
\hline & 33.33 & .00 & 20.00 & 34.62 & 18.18 & 20.00 & 26.03 \\
\hline \multirow[t]{3}{*}{ 500-999 Hours } & (2) & (0) & (0) & (2) & (1) & (0) & (5) \\
\hline & 40.00 & .00 & .00 & 40.00 & 20.00 & .00 & 100.00 \\
\hline & 13.33 & .00 & .00 & 7.69 & 9.09 & .00 & 6.85 \\
\hline \multirow[t]{3}{*}{ 1000+ Hours } & (4) & (3) & (6) & (6) & (4) & (1) & (24) \\
\hline & 16.67 & 12.50 & 25.00 & 25.00 & 16.67 & 4.17 & 100.00 \\
\hline & 26.67 & 50.00 & 60.00 & 23.08 & 36.36 & 20.00 & 32.88 \\
\hline \multirow[t]{3}{*}{ Total } & (15) & (6) & (10) & (26) & (11) & (5) & (73) \\
\hline & 20.55 & 8.22 & 13.70 & 35.62 & 15.07 & 6.85 & 100.00 \\
\hline & 100.00 & 100.00 & 100.00 & 100.00 & 100.00 & 100.00 & 100.00 \\
\hline
\end{tabular}


Table 1.5

Production Shut-down During Power Failure by Firm Size

\begin{tabular}{|c|c|c|c|c|c|c|c|}
\hline \multirow{2}{*}{$\begin{array}{l}\text { Hours Lost in } \\
1997\end{array}$} & \multicolumn{6}{|c|}{ Establishment Employment Size } & \multirow[b]{2}{*}{ Total } \\
\hline & $1-49$ & $50-99$ & $100-199$ & $200-499$ & $500-999$ & $1000+$ & \\
\hline \multirow[t]{3}{*}{ Zero Hours } & (3) & (1) & (0) & (6) & (4) & (3) & $(17)$ \\
\hline & 17.65 & 5.88 & .00 & 35.29 & 23.53 & 17.65 & 100.00 \\
\hline & 20.00 & 16.67 & .00 & 23.08 & 36.36 & 60.00 & 23.29 \\
\hline \multirow[t]{3}{*}{ 100-199 Hours } & (1) & (2) & (2) & (3) & (0) & (0) & (8) \\
\hline & 12.50 & 25.00 & 25.00 & 37.50 & .00 & .00 & 100.00 \\
\hline & 6.67 & 33.33 & 20.00 & 11.54 & .00 & .00 & 10.96 \\
\hline \multirow[t]{3}{*}{ 200-499 Hours } & (5) & (0) & (2) & (9) & (2) & (1) & (19) \\
\hline & 26.32 & .00 & 10.53 & 47.37 & 10.53 & 5.26 & 100.00 \\
\hline & 33.33 & .00 & 20.00 & 34.62 & 18.18 & 20.00 & 26.03 \\
\hline \multirow[t]{3}{*}{ 500-999 Hours } & (2) & (0) & (0) & (2) & (1) & (0) & (5) \\
\hline & 40.00 & .00 & .00 & 40.00 & 20.00 & .00 & 100.00 \\
\hline & 13.33 & .00 & .00 & 7.69 & 9.09 & .00 & 6.85 \\
\hline \multirow[t]{3}{*}{$1000+$ Hours } & (4) & (3) & (6) & (6) & (4) & (1) & (24) \\
\hline & 16.67 & 12.50 & 25.00 & 25.00 & 16.67 & 4.17 & 100.00 \\
\hline & 26.67 & 50.00 & 60.00 & 23.08 & 36.36 & 20.00 & 32.88 \\
\hline \multirow[t]{3}{*}{ Total } & (15) & (6) & (10) & (26) & (11) & (5) & (73) \\
\hline & 20.55 & 8.22 & 13.70 & 35.62 & 15.07 & 6.85 & 100.00 \\
\hline & 100.00 & 100.00 & 100.00 & 100.00 & 100.00 & 100.00 & 100.00 \\
\hline
\end{tabular}

shut-down during periods of power failure. Table 1.6 (Damage to Machinery and Equipment Due to Power Failure), shows that approximately 43 percent of the firms reported damage to their machinery and equipment and that 20 percent of these were small sized establishments. In Table 1.7 (Damage to Raw Materials Due to Power Loss by Firm Size), it is observed that 38 percent of the firms experienced damage to raw materials due to power failures. Of these 32 percent were small sized firms. Finally, Table 1.8 (Perceived Business Prospects by Firm Size), shows the firms' evaluation of their 'business prospects' and of those that reported declining prospects, 45 percent were firms with less than 50 employees. The electricity problem was cited as one of the critical factors contributing to the growth difficulties of most of the firms.

From Tables 1.2 through 1.7 it can be observed that small firms generally carry a higher burden of power supply failures. This has an important implication for the growth of industries and the generation of employment in urban areas of developing countries like Pakistan. Studies on regional patterns of industrial location in Bogota [Lee (1989)] and Seoul [Lee (1985)], show that small new firms tend to spend their early years near an urban centre where essential infrastructure services are easily accessed. The firms follow this pattern as it is expensive for them to operate independently in outlying areas where such infrastructure is unavailable. The studies show that these urban areas serve an 'incubator function' for small new firms which, as they become more independent, move out of the central area in search of more 
Table 1.6

Damage to Machinery and Equipment Due to Power Failure

\begin{tabular}{|c|c|c|c|c|c|c|c|}
\hline \multirow{2}{*}{$\begin{array}{l}\text { Hours Lost in } \\
1997\end{array}$} & \multicolumn{6}{|c|}{ Establishment Employment Size } & \multirow[b]{2}{*}{ Total } \\
\hline & $1-49$ & $50-99$ & $100-199$ & $200-499$ & $500-999$ & $1000+$ & \\
\hline \multirow[t]{3}{*}{ Zero Hours } & (3) & (1) & (0) & (6) & (4) & (3) & $(17)$ \\
\hline & 17.65 & 5.88 & .00 & 35.29 & 23.53 & 17.65 & 100.00 \\
\hline & 20.00 & 16.67 & .00 & 23.08 & 36.36 & 60.00 & 23.29 \\
\hline \multirow[t]{3}{*}{ 100-199 Hours } & (1) & (2) & (2) & (3) & $(0)$ & $(0)$ & $(8)$ \\
\hline & 12.50 & 25.00 & 25.00 & 37.50 & .00 & .00 & 100.00 \\
\hline & 6.67 & 33.33 & 20.00 & 11.54 & .00 & .00 & 10.96 \\
\hline \multirow[t]{3}{*}{ 200-499 Hours } & (5) & $(0)$ & (2) & (9) & (2) & (1) & $(19)$ \\
\hline & 26.32 & .00 & 10.53 & 47.37 & 10.53 & 5.26 & 100.00 \\
\hline & 33.33 & .00 & 20.00 & 34.62 & 18.18 & 20.00 & 26.03 \\
\hline \multirow[t]{3}{*}{ 500-999 Hours } & (2) & $(0)$ & $(0)$ & (2) & (1) & $(0)$ & $(5)$ \\
\hline & 40.00 & .00 & .00 & 40.00 & 20.00 & .00 & 100.00 \\
\hline & 13.33 & .00 & .00 & 7.69 & 9.09 & .00 & 6.85 \\
\hline \multirow[t]{3}{*}{$1000+$ Hours } & (4) & (3) & (6) & (6) & (4) & (1) & $(24)$ \\
\hline & 16.67 & 12.50 & 25.00 & 25.00 & 16.67 & 4.17 & 100.00 \\
\hline & 26.67 & 50.00 & 60.00 & 23.08 & 36.36 & 20.00 & 32.88 \\
\hline \multirow[t]{3}{*}{ Total } & (15) & (6) & (10) & (26) & (11) & (5) & (73) \\
\hline & 20.55 & 8.22 & 13.70 & 35.62 & 15.07 & 6.85 & 100.00 \\
\hline & 100.00 & 100.00 & 100.00 & 100.00 & 100.00 & 100.00 & 100.00 \\
\hline
\end{tabular}

Table 1.7

Damage to Raw Materials Due to Power Loss by Firm Size

\begin{tabular}{|c|c|c|c|c|c|c|c|}
\hline \multirow{2}{*}{$\begin{array}{l}\text { Hours Lost in } \\
1997\end{array}$} & \multicolumn{6}{|c|}{ Establishment Employment Size } & \multirow[b]{2}{*}{ Total } \\
\hline & $1-49$ & $50-99$ & $100-199$ & $200-499$ & $500-999$ & $1000+$ & \\
\hline \multirow[t]{3}{*}{ Zero Hours } & (3) & (1) & $(0)$ & (6) & (4) & (3) & $(17)$ \\
\hline & 17.65 & 5.88 & .00 & 35.29 & 23.53 & 17.65 & 100.00 \\
\hline & 20.00 & 16.67 & .00 & 23.08 & 36.36 & 60.00 & 23.29 \\
\hline \multirow[t]{3}{*}{ 100-199 Hours } & (1) & (2) & (2) & (3) & (0) & (0) & (8) \\
\hline & 12.50 & 25.00 & 25.00 & 37.50 & .00 & .00 & 100.00 \\
\hline & 6.67 & 33.33 & 20.00 & 11.54 & .00 & .00 & 10.96 \\
\hline \multirow[t]{3}{*}{ 200-499 Hours } & (5) & $(0)$ & (2) & (9) & (2) & (1) & (19) \\
\hline & 26.32 & .00 & 10.53 & 47.37 & 10.53 & 5.26 & 100.00 \\
\hline & 33.33 & .00 & 20.00 & 34.62 & 18.18 & 20.00 & 26.03 \\
\hline \multirow[t]{3}{*}{ 500-999 Hours } & (2) & (0) & (0) & (2) & (1) & (0) & (5) \\
\hline & 40.00 & .00 & .00 & 40.00 & 20.00 & .00 & 100.00 \\
\hline & 13.33 & .00 & .00 & 7.69 & 9.09 & .00 & 6.85 \\
\hline \multirow[t]{3}{*}{$1000+$ Hours } & (4) & (3) & (6) & (6) & (4) & (1) & (24) \\
\hline & 16.67 & 12.50 & 25.00 & 25.00 & 16.67 & 4.17 & 100.00 \\
\hline & 26.67 & 50.00 & 60.00 & 23.08 & 36.36 & 20.00 & 32.88 \\
\hline \multirow[t]{3}{*}{ Total } & (15) & (6) & (10) & (26) & (11) & (5) & (73) \\
\hline & 20.55 & 8.22 & 13.70 & 35.62 & 15.07 & 6.85 & 100.00 \\
\hline & 100.00 & 100.00 & 100.00 & 100.00 & 100.00 & 100.00 & 100.00 \\
\hline
\end{tabular}


Table 1.8

Perceived Business Prospects by Firm Size

\begin{tabular}{|c|c|c|c|c|c|c|c|}
\hline \multirow{2}{*}{$\begin{array}{l}\text { Business } \\
\text { Prospects }\end{array}$} & \multicolumn{6}{|c|}{ Establishment Employment Size } & \multirow[b]{2}{*}{ Total } \\
\hline & $1-49$ & $50-99$ & $100-199$ & $200-499$ & $500-999$ & $1000+$ & \\
\hline Rapidly & (2) & (1) & (2) & (4) & (1) & (2) & (12) \\
\hline \multirow{2}{*}{ Expanding } & 16.67 & 8.33 & 16.67 & 33.33 & 8.33 & 16.67 & 100.00 \\
\hline & 13.33 & 16.67 & 20.00 & 15.38 & 9.09 & 40.00 & 16.44 \\
\hline Expanding & (4) & (5) & (4) & (16) & (8) & (3) & $(40)$ \\
\hline \multirow[t]{2}{*}{ Somewhat } & 10.00 & 12.50 & 10.00 & 40.00 & 20.00 & 7.50 & 100.00 \\
\hline & 26.67 & 83.33 & 40.00 & 61.54 & 72.73 & 60.00 & 54.79 \\
\hline \multirow[t]{3}{*}{ No Change } & (4) & (0) & (0) & (6) & (0) & (0) & $(10)$ \\
\hline & 40.00 & .00 & .00 & 60.00 & .00 & .00 & 100.00 \\
\hline & 26.67 & .00 & .00 & 23.08 & .00 & .00 & 13.70 \\
\hline \multirow[t]{3}{*}{ Declining } & (5) & $(0)$ & (4) & (0) & (2) & $(0)$ & (11) \\
\hline & 45.45 & .00 & 36.36 & .00 & 18.18 & .00 & 100.00 \\
\hline & 33.33 & .00 & 40.00 & .00 & 18.18 & .00 & 15.07 \\
\hline \multirow[t]{3}{*}{ Total } & (15) & (6) & (10) & (26) & (11) & (5) & (73) \\
\hline & 20.55 & 8.22 & 13.70 & 35.62 & 15.07 & 6.85 & 100.00 \\
\hline & 100.00 & 100.00 & 100.00 & 100.00 & 100.00 & 100.00 & 100.00 \\
\hline
\end{tabular}

space. The research results suggest that large cities like Karachi with poor infrastructure offer limited incubation opportunity to small new firms that have difficulty in setting up private power generation or other essential services. The burdens therefore of inadequate public provision are especially severe for fledgling firms, which may start and grow in megacities like Karachi. This negative impact on the birth and growth of small firms will inhibit the generation of employment and income in megacities like Karachi. For example, the Bogota and Seoul studies also reveal that small firms generate 60 percent to 80 percent of the new job opportunities. This implies high returns of improved electricity service in specific areas where service reliability problems are user specific.

Future research could include samples of firms located in secondary cities such as Sialkot and Faisalabad. The comparison of the quality of infrastructure services between these cities and primate cities like Karachi would indicate the incubator function available for small firms in secondary cities. World Bank research [Anas et al. (1996)] on cities in Thailand and Indonesia concluded that inefficiencies in public power provision tend to be higher in secondary cities where the burdens on small firms are also greater.

\section{THE FIRMS' RESPONSES TO THE INEFFICIENCIES}

As shown in Table 1.1, the firms' responses are classified into four categories according to the sources of their power: (i) "KESC only" are captive firms that purchase all their electricity from KESC and therefore have no private investments to generate their own power; (ii) "KESC main" or stand-by firms rely on KESC but 
switch to their own generators during periods of breakdown in public supply; (iii) "OWN main" are those firms with the public supply as stand-by that normally use their own generators to ensure an adequate power supply, but switch to KESC during periods when the reliability of public power is considered high; and finally, (iv) "OWN only" are self-sufficient firms that have disconnected from the public grid.

In empirical studies [Anas and Lee (1989) and Anas et al. (1996)] on restructuring industrial organisation policy to facilitate infrastructure reform, it was observed that manufacturing firms used four ways to respond to inefficiencies in public electricity provision. Each response had its own economic rationale. The responses were (1) relocation, (2) factor substitution, (3) private provision, and (4) output reduction. In the interest of brevity, the third category, that was widely observed to occur in Karachi, is discussed. Within this category, four types of strategies are identified namely:

(a) Self-sufficiency: The firm provides its own electricity service to the point where there is no need for the public source. This strategy was observed mostly amongst large textile and garment manufacturers as well as multinational companies. Nearly all multinationals in the survey were selfsufficient in electricity provision. From Table 1.1, it can be gleaned that only 10 percent of the firms apply this strategy in electricity provision.

(b) Standby Private Provision: The firm has installed private generators and switches to these only when the reliability of KESC supply falls below a certain level. Table 1.1 shows that approximately 58 percent of the firms surveyed used this strategy as it appears to be the most popular mode of private provision across all firms and sectors.

(c) Public Source as Standby: The firm relies on private facility but switches to the KESC supply during those times of day when the public source is highly reliable. Again, from Table 1.1 it can be gleaned that nearly 10 percent of the surveyed firms reported such behaviour as public supply is not reliable.

(d) Captivity: The firm relies on KESC's network exclusively despite the very low reliability of service. This is the dominant strategy amongst small sized firms who cannot afford electricity generators. Nearly 24 percent of firms reported such behaviour with almost 41 percent being small sized firms.

The studies $(1989,1996)$ also suggest that there are economic incentives for three additional types of private provision regimes. These regimes are briefly discussed but they were not widely observed in Karachi because of extensive government restrictions on electricity trading between private firms. These regimes are (1) joint production, (2) satellite behaviour, and (3) shared production.

Joint production occurs when large firms invest substantially in private generation facilities and profit from selling part of the surplus output to neighbouring firms. Satellite behaviour is the opposite side of joint production and applies to those 
firms that buy electricity from other firms that produce surplus quantities. When a power interruption occurs, a satellite firm switches from the public source to the supply source of the nearby private producer.

The survey observed two cases of satellite behaviour, one in the garment and the second in the textile manufacturing sectors. In each case, a firm was buying surplus power from a neighbouring larger firm and the purchase was based on a contractually agreed price for fixed quantities of daily supply. In both cases, electricity was being traded without government approval. The manufacturers explained that it was too complicated to obtain a licence under the present regulatory system. The present regulatory framework is very stringent on electricity trading, which can take place only between private firms and public utilities and not between private firms themselves. Logic suggests that additional cases of satellite behaviour exist in Karachi because of the economic incentives involved in such a regime of private provision.

Finally, shared production concerns instances where several firms come together to form 'utility pools' or cartels to share the capital cost of private electricity generation by building facilities in industrial estates. There is anecdotal evidence of such behaviour in specific industrial sectors, such as textiles and chemicals in Lahore and Faisalabad, but such evidence was lacking in the sample survey for Karachi.

\section{THE CAPITAL COSTS OF PRIVATE PROVISION AND WILLINGNESS TO PAY FOR RELIABILITY}

The survey provides an illuminating study of the incidence of private provision, which is clearly the dominant response among Karachi's manufacturing firms. The survey yields important information concerning the average current market values of electric equipment and facilities used for private provision, and their share in the total value of the firms' machinery and equipment. The findings show that the average current market value of private power generation equipment for all firms is approximately Rs 10.7 billion which represents 11 percent of the total value of the firms' machinery and equipment. Furthermore, private power as an input has a 5 percent share of the variable cost of all inputs. The findings also reveal that nearly all firms in the stand-by regime have installed generation capacity sufficient to power an entire plant if KESC supply fails. In the stand-by firms approximately 37 percent of total power used during 1997 came from private generators and the remaining from KESC. If the typical installed private generator is sufficient to run an entire plant, then nearly 63 percent of the generation capacity of private generators remains idle or is under utilised. This idle capacity yields high total average cost of private power generation, especially when the costs of under utilisation and 100 percent utilisation are compared. The high cost of private provision sustained by these firms is the implicit value of service reliability that firms are willing to pay for. 
'Willingness to pay' is determined by calculating the average cost (per kWh) of electricity produced by the firms' private generators at the under utilised rate. The conclusion is that when the average cost of private provision is greater than the KESC price, the difference between these two prices yields the premium firms are willing to incur for reliable power supply.

In order to determine the average cost of private power generation, two sets of calculations are made by using the firms' reported power consumption from generators during 1997. In the first calculation, under utilisation reflects the cost of holding idle generating capacity, and in the second full utilisation assumes 100 percent generation from private source. The capital recovery cost is computed by annualising the current market value of the generators and accessories using the remaining service life. The recurring costs of fuel, labour and maintenance are added to the capital cost, and are duly adjusted to make pertinent calculations for the full utilisation case. Based on the actual average utilisation rate of 37 percent, the average cost per $\mathrm{kWh}$ is calculated as Rs 6.00, almost twice the present KESC tariff. Notably, under the 100 percent utilisation scenario the average cost per $\mathrm{kWh}$ falls substantially to Rs 2.00 .

In Table 2, the average fixed and variable costs of private power provision are calculated by size of firm. Small sized firms (1-49) show an average cost that is slightly lower than the sample mean. This is not because they are able to generate electric power at a lower cost, but because they cannot afford to make the expensive capital investment required for self-sufficiency. This group may be able to generate enough private power to support small-scale lighting and other basic infrastructure. Table 2.1 shows the premium for reliability for various representative scales of power production based on under utilisation. The unit premium is nearly twice over the public price for firms producing 100,000 to 2.5 million $\mathrm{kWh}$, declines for firms producing 5 million $\mathrm{kWh}$ annually, and virtually disappears for those producing 25 million or more $\mathrm{kWh}$ annually. This suggests that small users of electricity pay a much higher premium than large users. This further implies that if KESC improved its power reliability, it would yield greater savings to small firms than large ones.

In Table 2.2, a simple regression estimation is applied to calculate the cost function for various levels of private electricity production at 100 percent generation capacity. The scale of production is measured by the quantity of electricity (kWh) privately produced. To measure this, the annual cost of generation is computed. The annual cost has two components, (1) annualised fixed cost and (2) annualised variable cost, and the sum of (1) and (2) yields the annual total cost. The results show that the unit price paid declines with the quantity produced reflecting quantity discounts. Average cost functions are assumed to be negative exponential curves as they are expected to confirm the presence of strong economies of scale in private electricity production. The form of the negative exponential curve is the following:

$$
\text { (Average Cost) } i=A \exp (-a Q) e_{i} \quad \ldots \quad \ldots \quad \ldots
$$


Table 2

Average Fixed and Variable Costs of Private Power Generation per $\mathrm{kWh}$ by Size of Firm

\begin{tabular}{lccc}
\hline Firm Size & Fixed Cost* & Variable Cost** & Total \\
\hline $\begin{array}{l}\text { All Firms (Rs) } \\
\text { (Percent) }\end{array}$ & $\mathbf{3 . 2}$ & $\mathbf{3 . 1}$ & $\mathbf{6 . 3}$ \\
$1-49$ & 2.8 & 2.2 & 5.1 \\
$50-99$ & 0.8 & 1.2 & 2.0 \\
$100-199$ & 4.3 & 3.4 & 7.8 \\
$200-499$ & 2.6 & 3.5 & 6.1 \\
$500-999$ & 3.5 & 2.4 & 5.9 \\
1000 and Over & 1.0 & 2.1 & 2.8 \\
\hline $\begin{array}{l}\text { Note: For the sample firms as a whole, } 37 \text { percent of electric power supply came from private generators. } \\
\text { * Annualised capital value of generators and accessories. }\end{array}$ & \\
** Includes fuel, maintenance, parts, and labour. &
\end{tabular}

Table 2.1

Public and Private Unit Prices and Estimated Reliability Premia for Various Levels of Electricity Production

\begin{tabular}{lccc}
\hline $\begin{array}{c}\text { Q } \\
(1,000 \mathrm{kWh})\end{array}$ & $\begin{array}{c}\text { Public } \\
(\mathrm{Rs} / \mathrm{kWh})\end{array}$ & $\begin{array}{c}\text { Private } \\
(\mathrm{Rs} / \mathrm{kWh})\end{array}$ & $\begin{array}{c}\text { Premium for } \\
\text { Reliability }\end{array}$ \\
\hline 100 & 4.99 & 9.68 & 1.94 \\
1,000 & 4.60 & 7.08 & 1.53 \\
2,500 & 4.52 & 5.60 & 1.23 \\
5,000 & 3.93 & 4.01 & 1.02 \\
10,000 & 3.01 & 2.67 & 0.88 \\
25,000 & 2.67 & 1.90 & 0.71 \\
30,000 & 2.00 & 1.00 & 0.50 \\
\hline
\end{tabular}

Sources: Estimated Private Cost Function and Estimated KESC Tariff. Based on under utilisation. 
Table 2.2

Private Power Generation Cost Functions for Various Levels of Electricity Production (Fixed, Variable and Total Costs)

\begin{tabular}{lccc}
\hline $\begin{array}{c}\mathrm{Q} \\
(1,000 \mathrm{kWh})\end{array}$ & $\begin{array}{c}\text { Variable Cost } \\
(\mathrm{Rs} / \mathrm{kWh})\end{array}$ & $\begin{array}{c}\text { Fixed Cost } \\
(\mathrm{Rs} / \mathrm{kWh})\end{array}$ & $\begin{array}{c}\text { Total Cost } \\
(\mathrm{Rs} / \mathrm{kWh})\end{array}$ \\
\hline 50 & 3.81 & 4.19 & 8.26 \\
100 & 2.87 & 2.36 & 5.84 \\
500 & 1.49 & 0.62 & 2.61 \\
1,000 & 1.12 & 0.39 & 1.85 \\
1,500 & 0.95 & 0.25 & 1.51 \\
2,500 & 0.77 & 0.16 & 1.17 \\
3,500 & 0.67 & 0.12 & 0.99 \\
5,000 & 0.58 & 0.09 & 0.82 \\
10,000 & 0.44 & 0.05 & 0.58 \\
20,000 & 0.33 & 0.03 & 0.41 \\
25,000 & 0.30 & 0.02 & 0.37 \\
30,000 & 0.28 & 0.02 & 0.34 \\
\hline Sources: Estimated Private Cost Functions for Fixed, Variable and Total Costs based on 100 percent \\
generation.
\end{tabular}

where $i$ denotes the $i$ th firm in the sample. $A$ is a constant for the sample and $e$ is the multiplicative disturbance term of the average cost function, which varies from firm to firm in the sample of $i=1 \ldots .56$ firms which had generators. Therefore, the expectation is that $E\left(e_{i}\right)=1$. This cost function is linearised by taking the natural logarithm of both sides. Then,

$$
\ln [(\text { Average Cost }) i]=\ln A-a Q+\ln e_{i} \quad \ldots \quad \ldots \quad \ldots
$$

The linearised equation is estimated as a linear regression with the left side as the dependent variable, $\ln A$ as the intercept, $-a$ as the slope and $\ln e ;$ as a normal disturbance with $E\left[\ln e_{i}\right]=0$. This assumption on $\ln e_{i}$ implies that $E\left\{e_{i}\right\}=\exp$ $\left(s^{2} / 2\right)$, where $s$ is the standard error of the estimated regression. Then the average cost over the sample is:

$$
(\text { Average Cost })=\left[A \exp \left(s^{2} / 2\right)\right] \exp (-a Q) \quad \ldots \quad \ldots \quad \ldots
$$

The procedure is implemented three times, first for AFC, then for AVC, and finally for ATC. In each case the slopes are statistically significant, with the slope coefficient of the AATC function as -.05 and intercept as 4.07. The cost functions are transformed to the exponential form given by (3) and are plotted in Figure 1. A convenient way to evaluate the above cost functions is to use them to measure the 'output elasticity of average cost'. This elasticity is the percent decline in average cost $(\mathrm{Rs} / \mathrm{kWh})$ due to a one percent increase in the quantity of electricity produced 
है

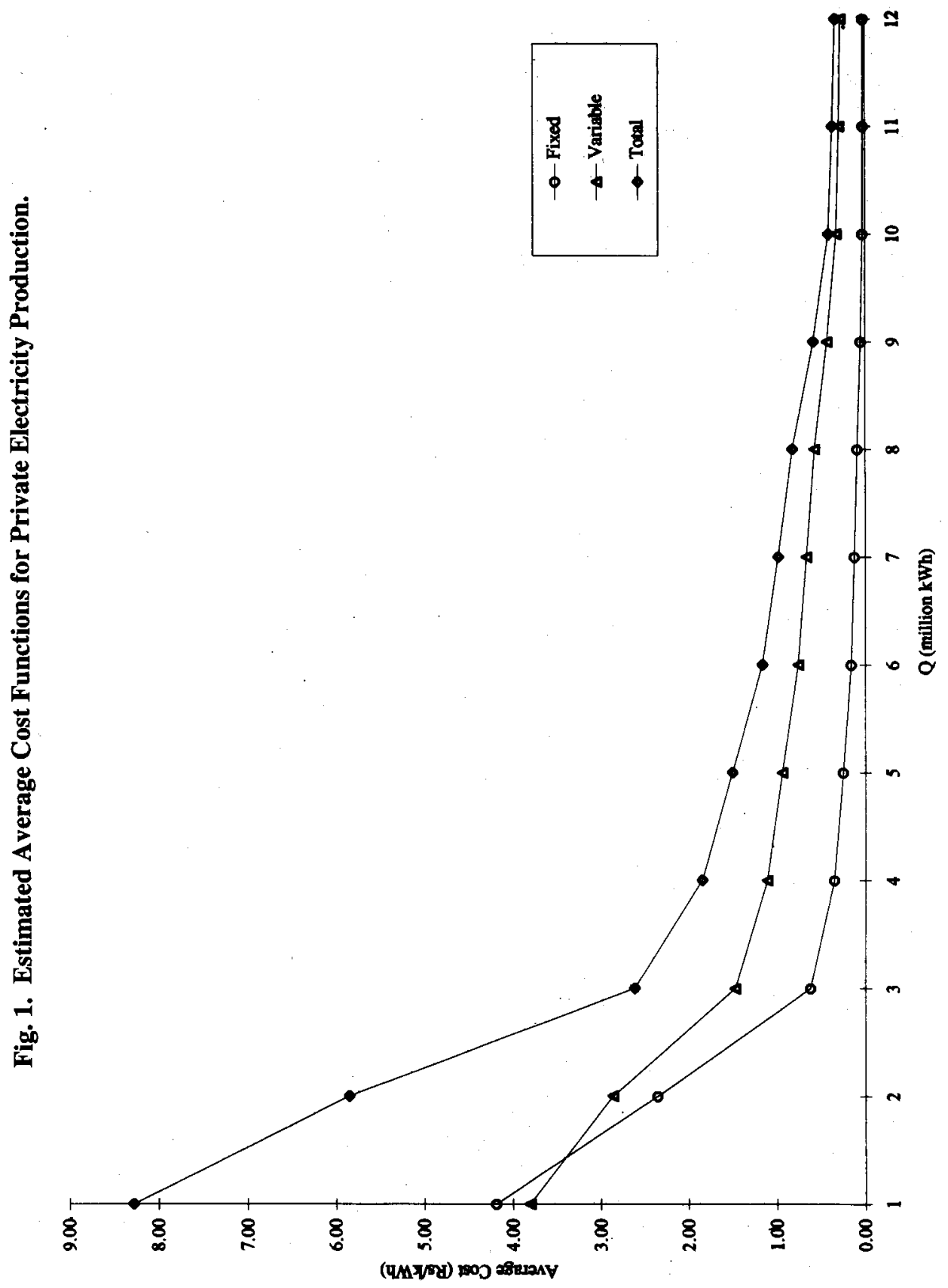


privately. The elasticity is $-a Q$. In the sample, the average quantity of electricity privately produced is approximately $4,233,000 \mathrm{kWh}$ per year. This number is used to evaluate the elasticity at the mean which shows that a 1 percent increase in private output results in a 0.05 percent reduction in the average cost of electricity. Since the elasticity increases linearly with level of electricity privately produced, the cost saving is greater for larger productions. For instance, in the sample, the mean quantity of electricity produced by the five largest producers is $219,360,600 \mathrm{kWh}$, or 50.82 times the mean quantity. The mean per unit cost for this sub-sample is Rs 6.19 $\mathrm{p} / \mathrm{kWh}$ rendering its elasticity to $(50.82 / 6.19)$ 8.2. The five largest firms therefore will realise an 8 percent saving in average cost by producing one percent more electricity. The conclusion: the more intensive a user of electricity, the stronger the incentive to produce larger quantity and to act as a joint producer of electricity. There are benefits in encouraging large manufacturers to contest KESC's monopoly as a producer and supplier of electricity to the industrial sector.

\section{POLICY IMPLICATIONS FOR IMPROVING ELECTRICITY PROVISION TO THE INDUSTRIAL SECTOR}

As discussed earlier, electricity provision in Pakistan suffers from two extreme cases of inefficiency: (a) a non-performing public sector with large capital investments, and (b) costly provision of the service by manufacturing firms. The private provision response observed in Karachi has developed over the decades due to chronic public sector failure. If this response had not emerged, the resulting welfare losses to society could have been much higher than they are today.

The current political-economic environment in Pakistan suggests that reforms in public sector performance will emerge gradually and be accompanied by major adjustments in pricing policy favouring higher tariffs. Although necessary for promoting allocative efficiency, the price changes will create burdens for industrial end-users. Hence the challenge is to identify intermediate policy options that can be easily implemented to bridge the gap between the two extreme inefficiencies and thus mitigate the burden on industrial users. The study suggests two intermediate policy options: (1) change the regulatory structure in Pakistan inducing greater utilisation of private provision capacities, especially in urban-industrial locations like Karachi; and (2) encourage greater private sector participation in the supply of electricity service to end-users.

Firstly, basic changes in the regulatory structure could benefit private firms. The current regulatory environment discourages the emergence of regimes such as joint production and shared production, which have been discussed earlier. If large firms with underutilised capacities are allowed to exploit economies of scale and compete with utilities like KESC, significant benefits can accrue to the industrial sector. KESC could raise upward its tariff rate for industrial users as they will have 
the choice to switch to private suppliers. Regulatory change will also encourage the establishment of 'utility pools' whereby firms could join hands to exploit economies of scale in the provision of electricity. The 'captive firms', which are mostly small ones, will realise benefits by joining the pool to become satellite firms for larger ones.

Secondly, the study suggests an industrial organisation policy that sets limits on protecting the public monopolies. Presently, the government in Pakistan (a) supports WAPDA and KESC as the only suppliers of power to end-users; (b) does not allow other private companies to enter the market for directly selling power to end-users; (c) levies a tax on imported power generators, and though firms are allowed to install their own generators, they can do so only by obtaining government approval; and (d) inter-firm power trading is restricted. Clearly the current policy toward encouraging competition in the electricity market is highly inefficient. It follows that by applying appropriate industrial organisation frameworks in the power sector, gains in economic efficiency and equity will be realised. For instance, recent literature [Mody (1996); Newbury (1995); Anas and Lee (1989)] re-examines competitive markets by arguing that an economic activity can be 'unbundled' into separate functions to enhance efficiency. For instance, functions that are subject to high sunk costs and scale economies, i.e., transmission and distribution, can remain in the public sector's hands. Those functions that are traditionally characterised by low entry costs, like power generation, can be made into a competitive industry. A fundamental policy aim in Pakistan's power generation should be to allow for a variety of market arrangements to emerge (utility pools, cartels) by liberalising the production, buying and selling of power among various users. This will induce large firms to utilise idle generating capacity by selling it to neighbouring firms and perhaps even to KESC, whose generation capacity has fallen short of increasing demand.

The most positive result of enhancing competition in the electricity market in megacities like Karachi will be that end-users have multiple sources for buying power. Consequently, the demand for public power, which is poor in quality and quantity, will diminish. Such policies are a simple but efficient way of dealing with the allocation of core infrastructure goods like electricity, which is critical for the productivity of a developing nation like Pakistan where the current discourse on public policy had better examine these options before implementing radical measures like outright privatisation.

\section{REFERENCES}

Anas, A., and K. S. Lee (1989) Infrastructure Investment and Productivity: The Case of Nigerian Manufacturing. A Framework for Policy Study. Review of Urban and Regional Development Studies. July. 
Anas, A., K. S. Lee, and G-T Oh (1996) Costs of Infrastructure Deficiencies in Manufacturing in Indonesia, Nigeria, and Thailand. The World Bank. (Policy Research Working Paper No. 1604.)

Batten, D. F. (1996) Infrastructure and the Complexity of Economic Development. Berlin; New York: Springer.

Baumol, W. J., and K. S. Lee (1991) Contestable Markets, Trade and Development. The World Bank Research Observer 38: Supplement, 9-36.

Baumol, W. J., J. Panzar, and R. D. Willig (1982) Contestable Markets and the Theory of Industry Structure. New York: Harcourt Brace Jovanovich.

Clarke, T., and C. Pitelis (eds) (1994) The Political Economy of Privatisation. Routledge: London and New York.

Kessides, C. (1993) Institutional Options for the Provision of Infrastructure. The World Bank, Washington, D. C. (World Bank Discussion Paper No. 212.)

Lee, K. S. (1985) An Evaluation of Decentralisation Policies in Light of Changing Location Patterns of Employment in the Seoul Region. The World Bank. (Urban Development Discussion Paper, UDD-60.)

Lee, K. S. (1989) The Location of Jobs in a Developing Metropolis: Patterns of Growth in Bogota and Cali, Colombia. Oxford: Oxford University Press.

Mody, A. (1996) Infrastructure Delivery, Private Interest and Public Good. Washington, D. C.: The World Bank.

Newbury, David (1995) A Template for Power Reform. Public Policy for the Private Sector. The World Bank, September.

Pasha, H. A., A. Ghaus, and S. Malik (1990) The Differential Impact of Power Loadshedding on Industrial Units in Pakistan. Pakistan Journal of Applied Economics 9:2 117-146.

Pollitt, M. G. (1995) Ownership and Performance in Electric Utilities: The International Evidence on Privatisation and Efficiency. Oxford Institute for Energy Studies, Oxford: Oxford University Press.

Sclar, Elliott D. (2000) You Don't Always Get What You Pay For: The Economics of Privatisation. Ithaca and London: Cornell University Press.

Vickers, J., and G. Yarrow (1997) Privatisation: An Economic Analysis. The MIT Press. 